\title{
Thermal and Chemical Stability of Two Homologous POZ/BTB Domains of KCTD Proteins Characterized by a Different Oligomeric Organization
}

\author{
Luciano Pirone, ${ }^{1}$ Carla Esposito, ${ }^{2,3}$ Stefania Correale, ${ }^{4}$ Giuseppe Graziano, ${ }^{5}$ \\ Sonia Di Gaetano, ${ }^{3}$ Luigi Vitagliano, ${ }^{3}$ and Emilia Pedone ${ }^{3}$ \\ ${ }^{1}$ Istituto di Cristallografia, CNR, Via G. Amendola 122/O, 70126 Bari, Italy \\ ${ }^{2}$ DFM Scarl, Via Mezzocannone 16, 80134 Napoli, Italy \\ ${ }^{3}$ Istituto di Biostrutture e Bioimmagini, CNR, Via Mezzocannone 16, 80134 Napoli, Italy \\ ${ }^{4}$ Kedrion S.p.A, S. Antimo, 80029 Napoli, Italy \\ ${ }^{5}$ Dipartimento di Scienze per la Biologia, la Geologia e l’Ambiente, Università del Sannio, Via Port'Arsa 11, 82100 Benevento, Italy
}

Correspondence should be addressed to Luigi Vitagliano; luigivitagliano@unina.it and Emilia Pedone; empedone@unina.it

Received 26 June 2013; Revised 11 September 2013; Accepted 18 September 2013

Academic Editor: D. M. Clarke

Copyright (C) 2013 Luciano Pirone et al. This is an open access article distributed under the Creative Commons Attribution License, which permits unrestricted use, distribution, and reproduction in any medium, provided the original work is properly cited.

POZ/BTB domains are widespread modules detected in a variety of different biological contexts. Here, we report a biophysical characterization of the POZ/BTB of KCTD6, a protein that is involved in the turnover of the muscle small ankyrin-1 isoform 5 and, in combination with KCTD11, in the ubiquitination and degradation of HDACl. The analyses show that the domain is a tetramer made up by subunits with the expected $\alpha / \beta$ structure. A detailed investigation of its stability, carried out in comparison with the homologous pentameric POZ/BTB domain isolated from KCTD5, highlights a number of interesting features, which are shared by the two domains despite their different organization. Their thermal/chemical denaturation curves are characterized by a single and sharp inflection point, suggesting that the denaturation of the two domains is a cooperative two-state process. Furthermore, both domains present a significant content of secondary structure in their denatured state and a reversible denaturation process. We suggest that the ability of these domains to fold and unfold reversibly, a property that is somewhat unexpected for these oligomeric assemblies, may have important implications for their biological function. Indeed, these properties likely favor the formation of heteromeric associations that may be essential for the intricate regulation of the processes in which these proteins are involved.

\section{Introduction}

A large fraction of proteins are oligomeric in their functional state(s). Although protein oligomerization may be incidental, several studies have highlighted the benefits of this process in a variety of different systems. In this framework, the analysis of the structural determinants of protein oligomerization is a field of considerable interest. The characterization of homologous proteins endowed with different quaternary structures is particularly suited to unveil key factors in protein oligomerization $[1,2]$.

Structural and functional literature studies have highlighted that specific domains, often shared by proteins involved in completely different biological processes, have been specifically designed by evolution to mediate proteinprotein interactions and to promote oligomerization. One of the best characterized domains deputed to these roles is the POZ/BTB domain, a motif that is widespread among eukaryotes [3]. From the structural point of view, this domain has a well-defined tridimensional fold characterized by a large interaction interface, that facilitates intermolecular interactions. This external surface is highly modifiable through amino-acid substitutions; indeed, variations on this common theme lead to a variegate ensemble of either transient or firm protein associations.

Among the proteins embedding POZ/BTB domain(s), the family denoted as KCTD, containing the potassium channel tetramerization domain, has recently received a 
special attention. Although the name of the family derives from their homology with proteins involved in the formation of voltage-gated $\mathrm{K}^{+}(\mathrm{Kv})$ channels, the twenty-three KCTD genes present in the human genome encode for proteins implicated in a variety of biological processes not connected with voltage channels. Recent investigations have shown that some members of the family play a key role in protein ubiquitination and degradation as they act as cullin binding adaptors (KCTD11, KCTD21, KCTD6, KCTD13, and KCTD10) [4-7]. In other members of the family, such as KCTD5, KCTD1, and KCTD7, that are known to bind cullin 3 , are likely involved in similar processes [8-10]. Finally, some KCTDs (KCTD8, KCTD12, KCTD12b, and KCTD16) are integral constituents of native GABAB receptors [11]. KCTDs are involved in the insurgence and the progression of severe human pathologies including cancers, epilepsy and obesity [12-14]. Despite their biological relevance, the molecular and structural characterization of these proteins is still limited.

Structural information have been so far reported only for KCTD5, KCTD11 and KCTD12 [6, 15-17]. KCTD5, the sole member of the family whose three-dimensional structure has been experimentally determined, presents a pentameric association of both the POZ/BTB N-terminal and the Cterminal domains [16]. A biophysical characterization of KCTD11 has shown that the protein and its N-terminal POZ/BTB domain are tetrameric. This observation is rather surprising considering the high sequence identity of KCTD11 and KCTD5 POZ/BTB (KCTD5 ${ }_{\mathrm{BTB}}$ ) domains and that evolutionary transitions from tetrameric (point symmetry $\mathrm{C} 4$ ) to pentameric (point symmetry C5) associations are very unlikely [1]. In order to gain further insights into the puzzling organization of POZ/BTB domains of KCTD proteins, we report the biophysical characterization of the POZ/BTB of KCTD6 (KCTD6 ${ }_{\text {BTB }}$ ), a member of the family that shares significant sequence and functional similarities with the well-characterized KCTD11. KCTD6 is a recently discovered substrate adaptor for cullin-3 that regulates protein levels of the muscle small ankyrin-1 isoform 5 (sAnk1.5) [18] and that, in combination with KCTD11, is also involved in ubiquitination and degradation of HDAC1, which is involved in the regulation of the acetylation state of the transcription factors Gli1 and Gli2 $[4,5,19]$. We then compared the thermal and the chemical stability of this tetrameric protein with the pentameric homolog POZ/BTB of KCTD5. Interestingly, both proteins exhibit reversible denaturation despite their oligomeric organization.

\section{Materials and Methods}

2.1. Cloning, Expression and Purification of $\mathrm{KCTD}_{B T B}$ and KCTD $5_{\text {BTB }}$. KCTD6 $6_{\text {BTB }}$ (residues 10-110 of KCTD6) was amplified by PCR, using as template the human KCTD6 cDNA (NM_153331) (thermo Scientific) and cloned into the pETM-11 expression vector (Novagen). Escherichia coli BL21(DE3) Star strain (Invitrogen) was transformed with the recombinant vector, grown at $37^{\circ} \mathrm{C}$ and induced by $0.5 \mathrm{mM}$ isopropyl- $\beta$-d-thiogalactoside (IPTG) for $16 \mathrm{~h}$ at $22^{\circ} \mathrm{C}$.
The cell pellets were re-suspended in $50 \mathrm{mM}$ Tris- $\mathrm{HCl}$, $0.1 \mathrm{M} \mathrm{NaCl}, \mathrm{pH}$ 8.0. The KCTD6 ${ }_{\mathrm{BTB}}$ domain was purified as already described for KCTD11 with slight modifications [6].

The pET28/KCTD5 ${ }_{\text {BTв }}$ plasmid was a gift of Prof. Goldstein (University of Chicago). The recombinant protein was expressed and purified according to the procedure previously reported.

2.2. Static Light Scattering. Static light scattering experiments were performed using a MiniDAWN Treos spectrometer (Wyatt Instrument Technology Corp.) equipped with a laser operating at $658 \mathrm{~nm}$ and connected online to a size-exclusion chromatography.

Purified KCTD6 $_{\text {BTB }}$ was analyzed by size-exclusion chromatography connected to a triple-angle light scattering detector equipped with a QELS module (quasielastic light scattering) for mass value. $500 \mu \mathrm{L}$ sample $(1 \mathrm{mg} / \mathrm{mL})$ was loaded on a Superdex 75 10/30 column, equilibrated in $50 \mathrm{mM}$ Tris- $\mathrm{HCl}$ and $0.15 \mathrm{M} \mathrm{NaCl}, \mathrm{pH} 8.0$ and analyzed as described elsewhere [6].

2.3. Spectroscopic Studies. CD spectra were recorded at $20^{\circ} \mathrm{C}$ using a Jasco J-810 spectropolarimeter equipped with a Peltier thermostatic cell holder. Far-UV measurements (260$190 \mathrm{~nm}$ ) were carried out using a $0.1 \mathrm{~cm}$ path length cell in a $10 \mathrm{mM}$ Tris- $\mathrm{HCl}, \mathrm{pH} 8.0$ buffer at a concentration of $5 \mu \mathrm{M}$.

Chemical denaturation was induced by guanidine hydrochloride $(\mathrm{GuHCl})$ or urea in a range of 0 to $6.0 \mathrm{M}$ and 0 to $8.0 \mathrm{M}$, respectively, incubating the samples $2 \mathrm{~h}$ at room temperature. All the denaturations were investigated by recording the CD signal at $222 \mathrm{~nm}$.

Fluorescence experiments were conducted in the same conditions. Fluorescence spectra were collected at $20^{\circ} \mathrm{C}$ using a Varian Cary Eclipse spectrophotometer and a $1.0 \mathrm{~cm}$ path length cell. For KCTD $5_{\mathrm{BTB}}$, two separate sets of experiments were carried out setting the excitation wavelength at either 280 or $295 \mathrm{~nm}$. For KCTD6 ${ }_{\mathrm{BTB}}$, that does not contain Trp residues, only the experiment with excitation at $280 \mathrm{~nm}$ was performed. In all cases, the emission was recorded in the range $290-450 \mathrm{~nm}$.

\section{Results}

3.1. Sequence Analysis and Secondary Structure Content of KCTD6. KCTD6 is a protein of 237 residues (UniProt code Q8NC69). Comparative analyses of its sequence with those reported in the sequence databanks clearly indicate that the POZ/BTB domain, located in the N-terminal region of the protein, namely $\mathrm{KCTD} 6_{\mathrm{BTB}}$, spans from residue 10 to residue 110. Pair-wise alignments of $\mathrm{KCTD}_{\mathrm{BTB}}$ with POZ/BTB domains of other KCTDs unveil that the closest ones are those of KCTD11 and KCTD21. The sequence identity shared by $\mathrm{KCTD}_{\mathrm{BTB}}$ and KCTD21 $1_{\mathrm{BTB}}$ with $\mathrm{KCTD}_{\mathrm{BTB}}$ is $61 \%$ and $72 \%$, respectively. $\mathrm{KCTD}_{\mathrm{BTB}}$ presents sequence identities higher than 50\% when compared with KCTD1 (54\%), KCTD4 (53\%), and KCTD15 (57\%).

To gain insights into the structural features of the protein, we performed a secondary structure prediction 


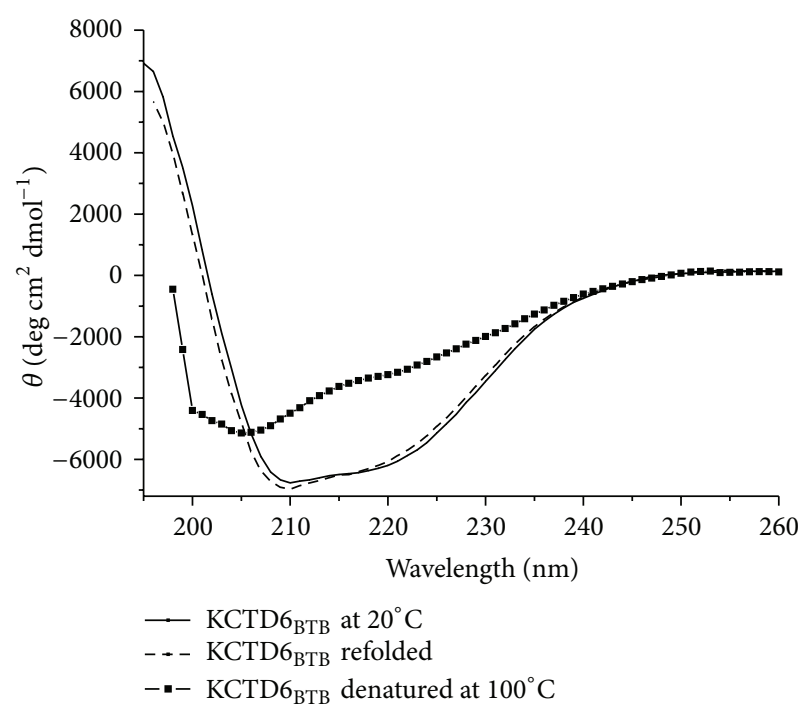

(a)

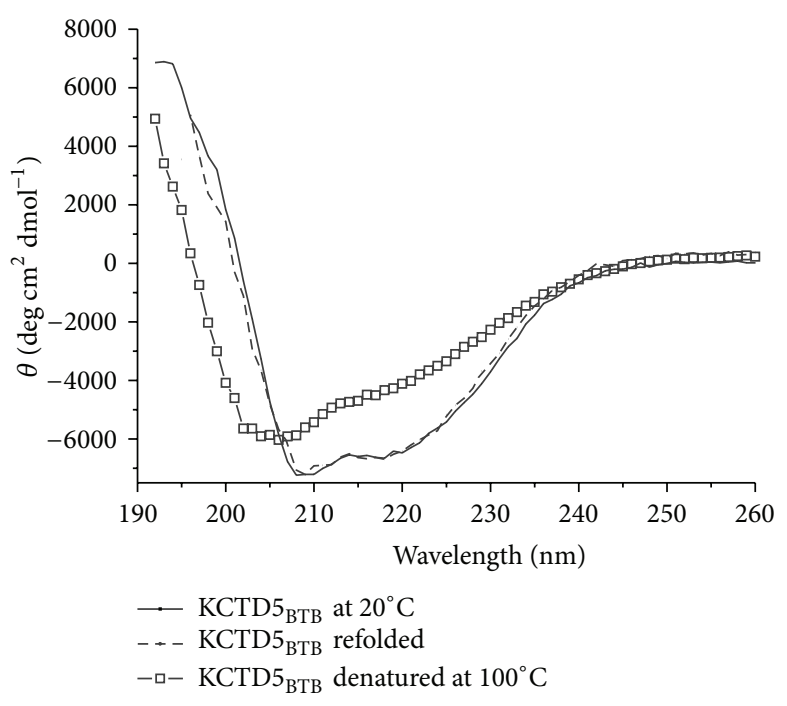

(b)

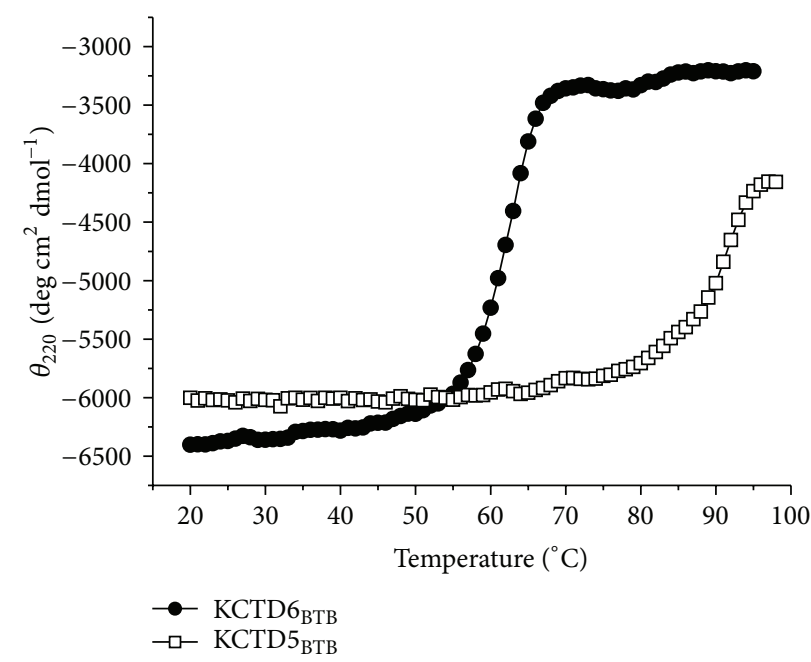

(c)

Figure 1: CD spectra of $\mathrm{KCTD}_{\text {Втв }}$ (a) and $\mathrm{KCTD}_{\mathrm{BTB}}$ (b) curves recorded at $20^{\circ} \mathrm{C}$, at $100^{\circ} \mathrm{C}$, and after decreasing the temperature back to $20^{\circ} \mathrm{C}$ are indicated with solid, dash, and dash-dot lines, respectively. Thermal denaturation curves are shown in (c).

session on KCTD6 sequence by using the Swiss Model server (http://swissmodel.expasy.org/). The server predicts the occurrence of several $\beta$-strands and $\alpha$-helices in KCTD structure (Figure S1) (Supplementary Material available online at: http://dx.doi.org/10.1155/2013/162674). The inspection of the region 10-110, corresponding to the BTB domain, unveils the presence of the structural elements (three $\beta$ strands and five $\alpha$-helices) characteristic of this motif. The prediction also suggests that the C-terminal domain of the protein is highly structured, being characterized by the combination of $\alpha$ - and $\beta$-structure with coil regions of limited size.

3.2. Expression and Characterization of $K C T D 6_{B T B}$ Oligomerization State. In order to perform a biophysical characterization of $\mathrm{KCTD}_{\mathrm{BTB}}$, this domain was cloned and expressed in E. coli. The particularly high yield $(\sim 1 \mathrm{~g} / \mathrm{L})$ of the purified recombinant product allowed its extensive biophysical characterization. The homogeneity of the purified protein was assessed with a variety of different techniques (SDS PAGE and mass spectrometry). The far-UV CD spectrum of KCTD6 ${ }_{\mathrm{BTB}}$ is consistent with a properly folded protein (Figure $1(\mathrm{a})$ ). The spectrum is characterized by the presence of two minima (at 210 and $219 \mathrm{~nm}$ ) and one maximum (at $195 \mathrm{~nm}$ ) which are typical fingerprints of $\alpha / \beta$ proteins.

As POZ/BTB domains of KCTD proteins may be characterized, despite their high sequence identities, by different oligomeric states, we carefully investigated the aggregation state of KCTD $_{\text {BTB }}$. Light scattering measurements provided a weight-average molar mass for $\mathrm{KCTD}_{\mathrm{BTB}}$ of $50580 \pm$ $350 \mathrm{Da}$ (Figure 2). These experiments suggest a tetrameric organization of the protein. This finding is in line with gel filtration experiments (Table S1). These results are not 


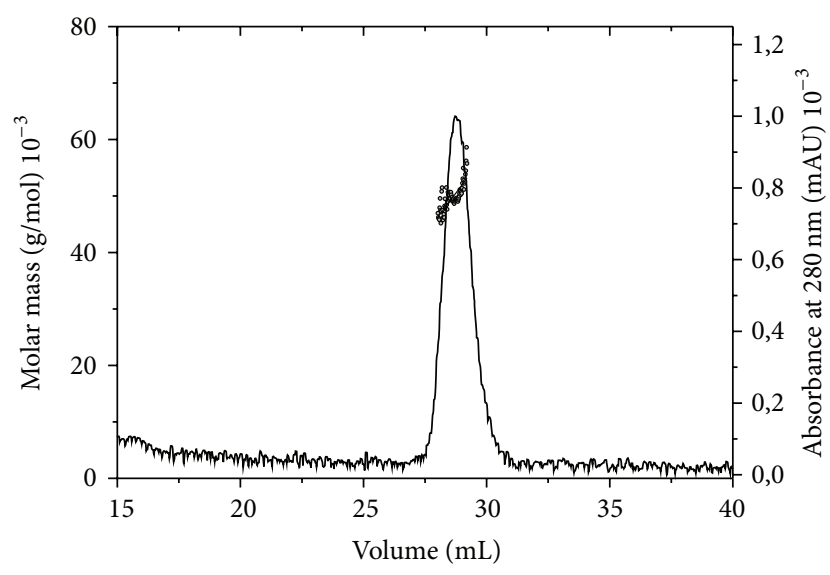

FIGURE 2: Static light scattering analysis (molar mass versus elution volume) of $\mathrm{KCTD}_{\mathrm{BTB}}$.

surprising as $\mathrm{KCTD}_{\mathrm{BTB}}$ shares a very high sequence identity with $\mathrm{KCTD}_{\mathrm{BTB}}(61 \%)$ and $\mathrm{KCTD}_{\mathrm{B}} \mathrm{1}_{\mathrm{BTB}}(72 \%)$, which are also tetrameric in solution [6]. It is also worth mentioning that the residues that stabilize the pentameric interface in KCTD5 are not conserved in KCTD6.

3.3. Thermal Denaturation: $K C T D 6_{\text {ВTB }}$ versus $K C T D 5_{B T B}$. Thermal denaturation analyses of the KCTD $6_{\text {Втв }}$ domain were conducted by using far-UV CD spectroscopy by following the CD signal at $222 \mathrm{~nm}$. As the tetrameric $\mathrm{KCTD}_{\mathrm{BTB}}$ shares a significant sequence identity (approx. 40\%) with the pentameric $\mathrm{KCTD}_{\text {BTв }}$ (Figure S2), the analyses were extended to the latter domain for comparative purposes. The far-UV CD spectrum of the KCTD $6_{\text {ВTВ }}$ sample kept at $100^{\circ} \mathrm{C}$ is different from that recorded for the native form at $20^{\circ} \mathrm{C}$, but is characterized by the presence of a broad minimum at $205 \mathrm{~nm}$ and a shoulder at $220 \mathrm{~nm}$ (Figure 1(a)). The latter features are indicative of the persistence of secondary structure elements in the thermally denatured form of the domain. Interestingly, when the temperature is decreased back to $20^{\circ} \mathrm{C}$, КСTD $6_{\text {ВТВ }}$ fully recovers its initial structure, as indicated by the far-UV CD spectrum (Figure 1(a)). This demonstrates that the thermal denaturation of $\mathrm{KCTD}_{\mathrm{BTB}}$ is a reversible process. As shown in Figure 1(c), the thermal denaturation curve of $\mathrm{KCTD}_{\mathrm{BTB}}$ presents a sigmoidal shape with a single inflection point, corresponding to a melting temperature $\left(T_{m}\right)$ of $62^{\circ} \mathrm{C}$, suggestive of a cooperative process in which dissociation and unfolding are coupled. The latter mechanism is supported by the fact that, on increasing the protein concentration, the melting curves present the same shape with an increase in the melting temperature (data not shown), as it should be for a reversible two-state process in which dissociation and unfolding are coupled [20,21].

Similar analyses conducted on KCTD $5_{\text {BTB }}$ unveil differences and analogies when compared to KCTD $6_{\text {Втв }}$. The overall shape of the far-UV CD spectrum of KCTD $5_{\mathrm{BTB}}$ is similar to that of KCTD6 $6_{\text {Втв }}$ (Figure 1(b) and Figure S3) suggesting an analogous secondary structure content, in line with the high sequence identity. As in the case of $\mathrm{KCTD}_{\text {втв: }}$ (a) the thermally-denatured state of $\mathrm{KCTD} 5_{\mathrm{BTB}}$ is characterized by the presence of a significant content of residual secondary structure and (b) the $\mathrm{KCTD}_{\mathrm{BTB}}$ thermal denaturation is also reversible, since the secondary structure is recovered when temperature is lowered to $20^{\circ} \mathrm{C}$ (Figure 1(b)). As shown in Figure $1(\mathrm{c})$, the thermal denaturation curve of $\mathrm{KCTD}_{\mathrm{BTB}}$ also presents a sigmoidal shape with a single inflection point, suggestive of a cooperative process in which dissociation and unfolding are coupled. However, CD measurements show that the pentameric $\mathrm{KCTD} 5_{\mathrm{BTB}}$ is significantly more stable than the tetrameric KCTD $6_{\mathrm{BTB}}$. Indeed, when the stability of $\mathrm{KCTD} 5_{\mathrm{BTB}}$ is measured using the same concentration used for $\mathrm{KCTD}_{\mathrm{BTB}}$, its $T_{m}$ value is as high as $93^{\circ} \mathrm{C}$ (Figure $1(\mathrm{c})$ ). Therefore, reversibility and cooperativity of the temperatureinduced denaturation (i.e., a reversible two-state process in which dissociation and unfolding are coupled) is a common feature of KCTD5 $5_{\text {Втв }}$ and $\mathrm{KCTD}_{\text {Втв}}$, despite their complex and diversified oligomeric organization.

3.4. Chemical Denaturation: $K C T D 6_{B T B}$ versus $K C T D 5_{\text {ВTB }}$. The comparative analysis of the conformational stability of $\mathrm{KCTD}_{\mathrm{BTB}}$ and $\mathrm{KCTD} 5_{\mathrm{BTB}}$ was extended by performing chemical denaturation experiments using either urea or $\mathrm{GuHCl}$ as denaturant and the same protein concentration. To gain insights into these events at the level of both secondary and tertiary structure, the conformational stability of the two domains against the denaturing action of urea and $\mathrm{GuHCl}$ was investigated by recording (a) the change in molar ellipticity at $222 \mathrm{~nm}$ and (b) the change in fluorescence spectrum upon excitation at either $280 \mathrm{~nm}$ or $295 \mathrm{~nm}$. The evolution of the CD signal at $222 \mathrm{~nm}$ upon the addition of the denaturants is characterized by a sigmoidal shape, with a single inflection point (Figure 3), and the denaturation proves to be reversible for both proteins against both denaturants. The transition is sharper upon the addition of $\mathrm{GuHCl}$, although the process does appear to be rather cooperative for both denaturants. Despite the differences exhibited in the thermal denaturation analysis, the two domains are characterized by a roughly similar stability against the denaturing action of urea and $\mathrm{GuHCl}$, respectively. Indeed, the $\mathrm{C}_{1 / 2}$ value exhibited by the two domains upon addition of urea is $4.7 \mathrm{M}$ for $\mathrm{KCTD}_{\mathrm{BTB}}$ and $5.0 \mathrm{M}$ for $\mathrm{KCTD}_{\mathrm{BTB}}$. As expected, the $\mathrm{C}_{1 / 2}$ value, which is $2.2 \mathrm{M}$ for both proteins, is lower in the presence of $\mathrm{GuHCl}$. The non-zero CD signal at $222 \mathrm{~nm}$, observed at very high urea concentration, suggests that the urea-denatured state is characterized by a residual secondary structure content. This is particularly evident in the case of $\mathrm{KCTD}_{\text {Втв }}$. When $\mathrm{GuHCl}$ is used as denaturant, the content of residual secondary structure is marginal for both domains.

The intensity of the fluorescence spectra of the two domains shows clear variations when the concentration of the denaturant approaches the $\mathrm{C}_{1 / 2}$ values determined in the $\mathrm{CD}$ analysis (Figure 4). This observation strongly suggests that structural transitions at the secondary and tertiary level are concomitant and lead to a cooperative and reversible process. A comparative analysis of the fluorescence spectra of the two domains shows some significant differences. The variation of the intensity in the fluorescence spectra of $\mathrm{KCTD}_{\mathrm{BTB}}$, which 


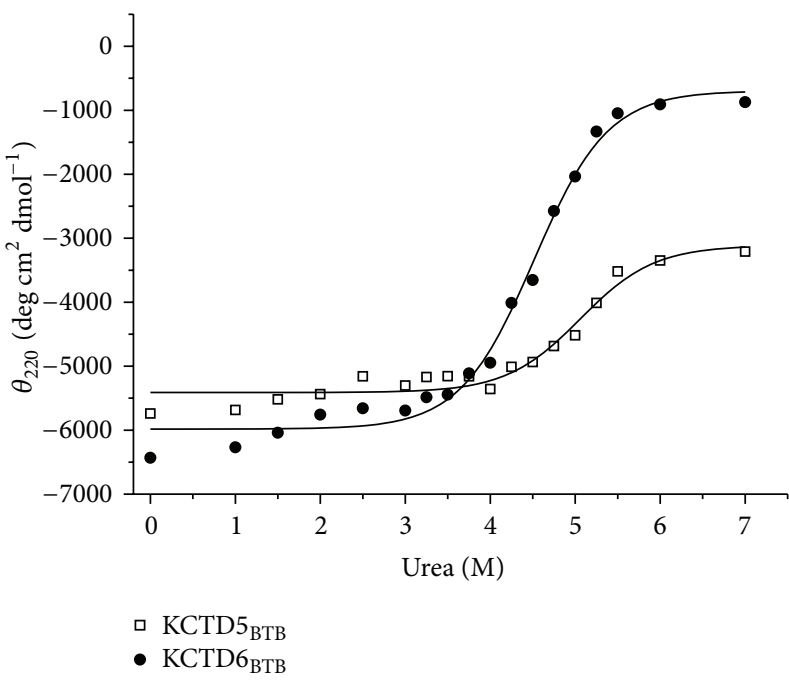

(a)

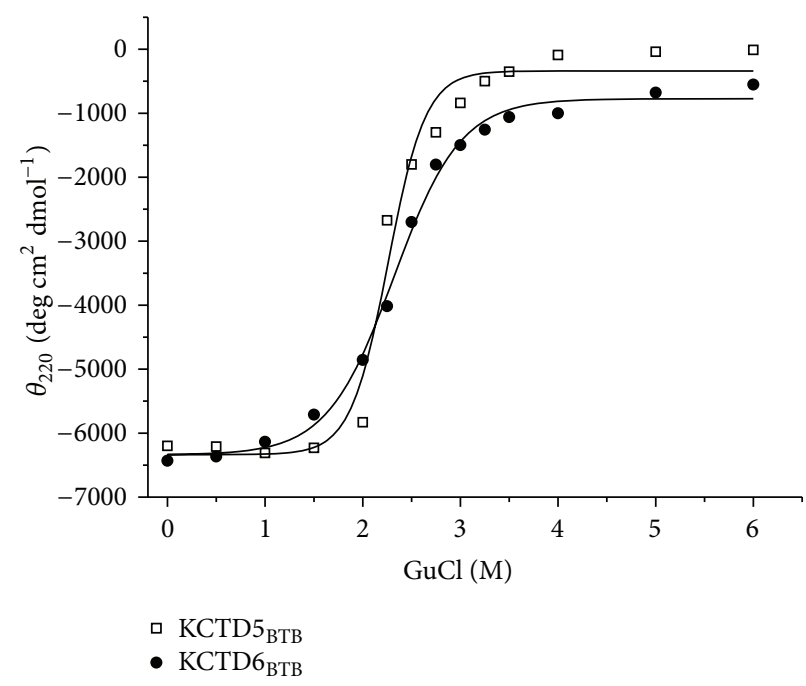

(b)

Figure 3: Chemical denaturation induced by urea (a) or $\mathrm{GuHCl}(\mathrm{b})$ of $\mathrm{KCTD}_{\mathrm{BTB}}$ and $\mathrm{KCTD} 5_{\mathrm{BTB}}$ followed by CD spectroscopy.

were recorded exclusively upon excitation at $280 \mathrm{~nm}$ is not coupled with changes in the wavelength of the maximum that remains close to $305 \mathrm{~nm}$. On the other hand, KCTD $5_{\mathrm{BTB}}$ fluorescence spectra collected in similar conditions are characterized by a red shift of the wavelength of the maximum, while the intensity decreases. Since KCTD $5_{\text {Втв }}$ sequence contains a single $\operatorname{Trp}$ residue (Trp45), fluorescence spectra were also collected upon excitation at $295 \mathrm{~nm}$. These spectra show trends similar to those recorded upon excitation at $280 \mathrm{~nm}$. The addition of both denaturants produces a decrease of the intensity and a shift of the wavelength of the maximum from 330 to $360 \mathrm{~nm}$. This finding indicates that the side chains of the five Trp present in KCTD $5_{\text {ВTв }}$ pentamer, that are buried in the native structure, become solvent exposed in the denatured state. Since Trp45 is located in the initial strand of the sole $\beta$-sheet present in $\mathrm{KCTD} 5_{\mathrm{BTB}}$ structure, this finding may suggest that the $\beta$-structure is highly perturbed in the denaturation and that the residual structure present in the denatured state corresponds to helical regions. It is also likely that the differences observed in the fluorescence spectra of the two proteins, recorded upon excitation at $280 \mathrm{~nm}$, may be due to the contribution of the Trp 45 side chains in the KCTD $5_{\text {ВTB }}$ domain.

\section{Discussion}

POZ/BTB domains are versatile modules that are often implicated in fundamental biological processes [22]. Previous literature data have shown that these domains are able to selfassociate and to form specific homo- and hetero-oligomers [22]. In this framework, the elucidation of the biophysical properties of these domains represents an important step for a full understanding of their role and for designing ad hoc strategies aimed at modulating their activities. Here, we report a characterization of two POZ/BTB domains
(KCTD6 ${ }_{\mathrm{BTB}}$ and $\left.\mathrm{KCTD} 5_{\mathrm{BTB}}\right)$ isolated from proteins belonging to the family of KCTDs, an emerging class of key factors involved in severe human pathologies. Although $\mathrm{KCTD}_{\mathrm{BTB}}$ and $\mathrm{KCTD} 5_{\mathrm{BTB}}$ share a high sequence identity $(>40 \%)$, these domains are characterized by a different oligomeric organization. Indeed, literature data have shown that $\mathrm{KCTD} 5_{\mathrm{BTB}}$ is pentameric $[6,16]$, whereas present data indicate that $\mathrm{KCTD}_{\mathrm{BTB}}$ is tetrameric. The tetrameric association of $\mathrm{KCTD}_{\mathrm{BTB}}$ is not surprising taking into account the high sequence identity $(>61 \%)$ that this domain shares with the tetrameric POZ/BTB domains of KCTD11 and KCTD21. Moreover, the analysis of $\mathrm{KCTD}_{\mathrm{BTB}}$ sequence indicates that this domain does not contain the residues that are believed to stabilize the pentameric association of KCTD5 $5_{\mathrm{BTB}}$ (Figure S2) [16]. The finding that these two domains adopt a different oligomeric organization, despite the high sequence homology, is even more puzzling considering that they play a similar biological function: the recruitment of cullin 3 in the E3 ligase complex $[4,18]$. It is important to note that from the evolutionary point of view, the transition in homologous proteins from cyclic tetrameric to cyclic pentameric states is a very rare (low probability) event [1].

Even though both domains display a remarkable stability against temperature, a significant difference is emerged: the melting temperature is $62^{\circ} \mathrm{C}$ for $\mathrm{KCTD}_{\mathrm{BTB}}$ but $93^{\circ} \mathrm{C}$ for KCTD5 $5_{\text {BTв }}$. Although the absence of structural data for KCTD6 $_{\text {BTB }}$ hampers the possibility to relate this marked difference to specific structural determinants, it is likely that the peculiar stability of $\mathrm{KCTD}_{\text {Втв }}$ may be related, among other factors, to the presence of an intrasubunit disulfide bridge that connects Cys62 to Cys74 [16]. In addition, the analysis of the $3 \mathrm{D}$ structure shows that each subunit is involved in extensive interactions in the pentameric organization. Indeed, approximately $25 \%$ of the surface of each subunit (about $1500 \AA^{2}$ out of $6000 \AA^{2}$ ) is buried upon pentamer formation. 

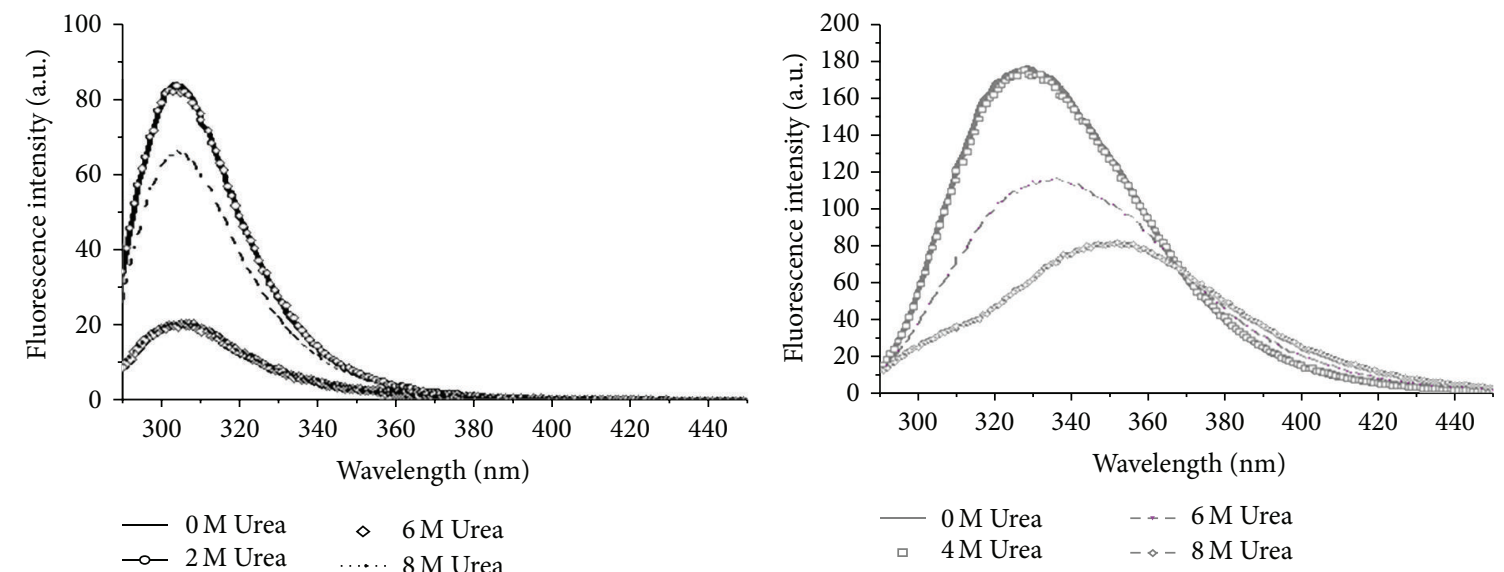

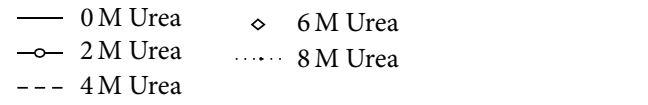

(a)

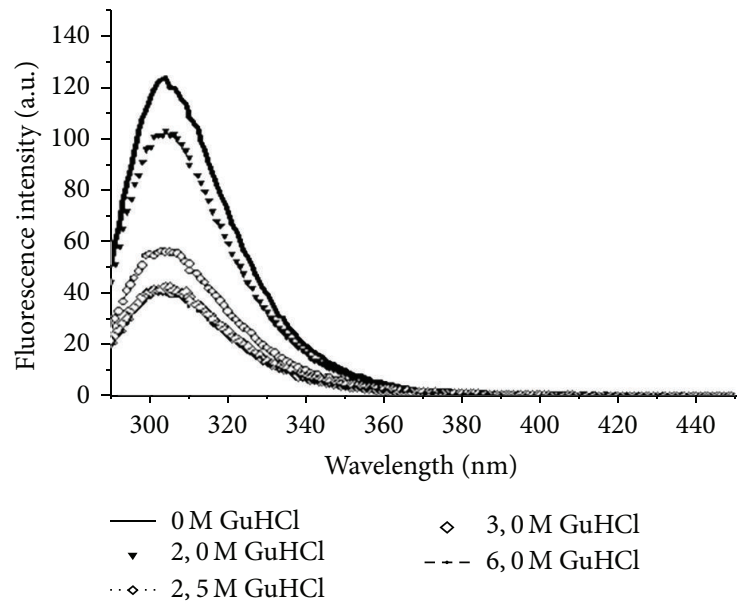

(c)

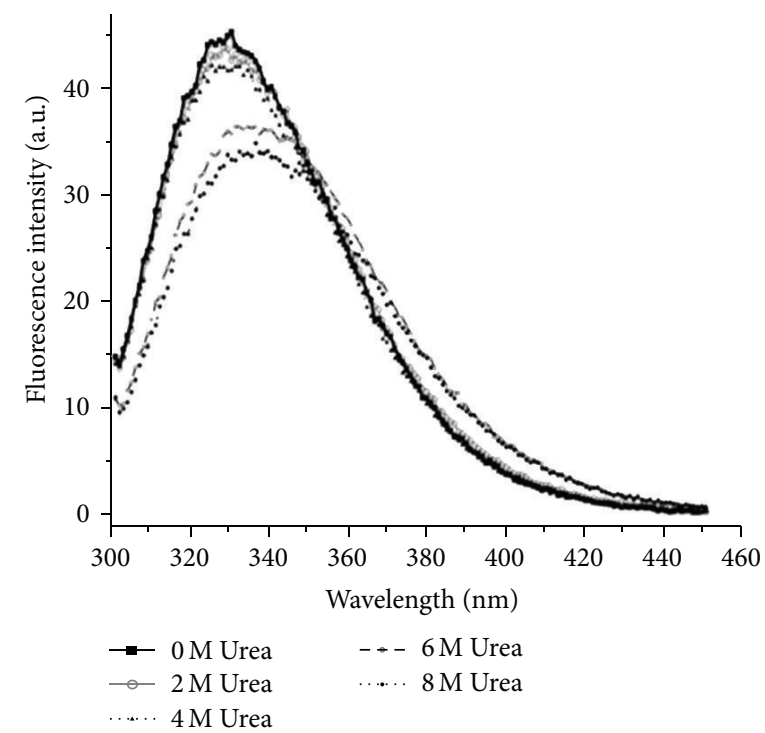

(e)

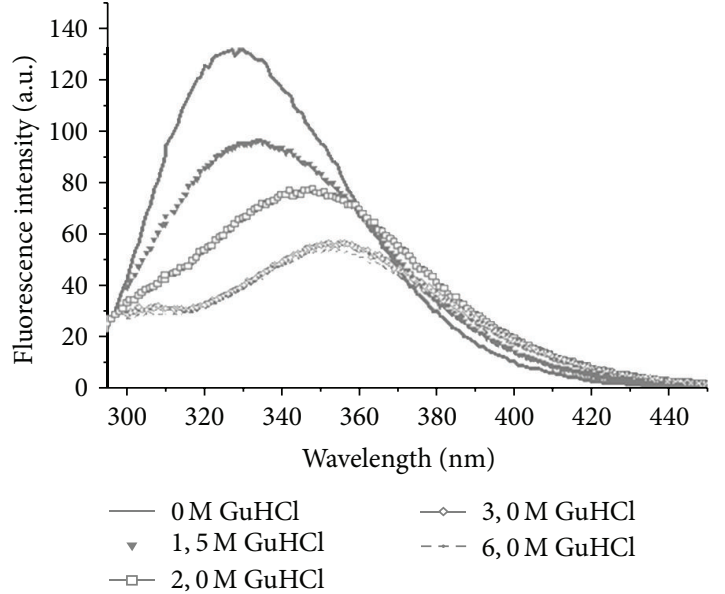

(d)

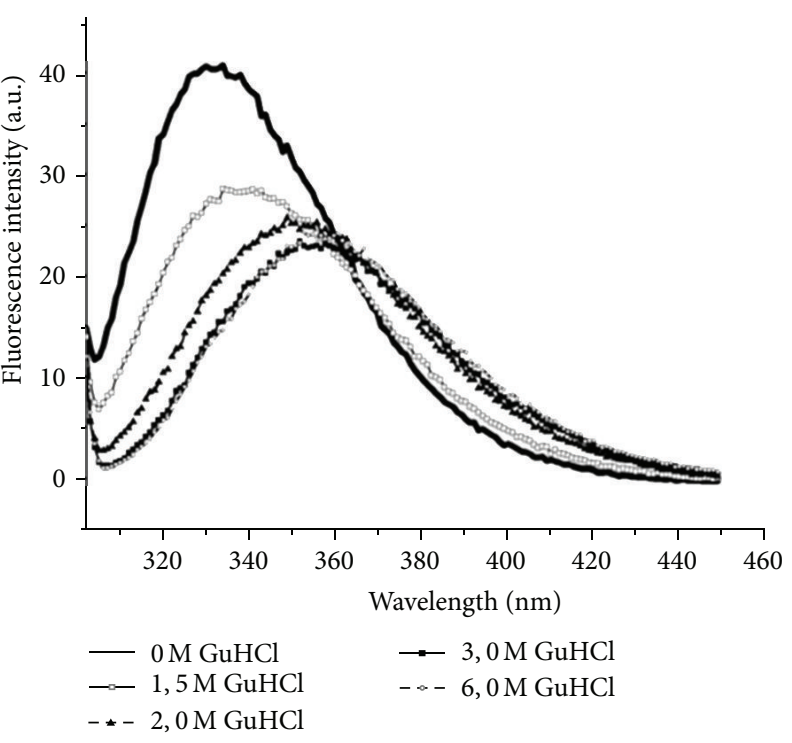

(f)

FIGURE 4: Chemical denaturation of $\mathrm{KCTD}_{\mathrm{BTB}}$ and $\mathrm{KCTD} 5_{\mathrm{BTB}}$ followed by fluorescence. The spectra obtained upon excitation at $280 \mathrm{~nm}$ in the presence of increasing concentration of urea are reported in (a) and (b) for KCTD6 $6_{\mathrm{BTB}}$ and $\mathrm{KCTD} 5_{\mathrm{BTB}}$, respectively. Similarly, the spectra obtained upon excitation at $280 \mathrm{~nm}$ in the presence of increasing concentration of $\mathrm{GuHCl}$ are reported in (c) and (d), for KCTD6 ${ }_{\text {BTB }}$ and $\mathrm{KCTD}_{\text {Втв }}$, respectively. Spectra obtained for KCTD5 ${ }_{\text {Втв }}$ after excitation at $295 \mathrm{~nm}$ are reported in (e) (urea) and (f) $(\mathrm{GuHCl})$. 
On the other hand, it is important to note that the stability of KCTD5 $5_{\mathrm{BTB}}$ against chemical denaturants (urea and $\mathrm{GuHCl}$ ) is less remarkable. $\mathrm{KCTD}_{\mathrm{BTB}}$ and $\mathrm{KCTD} 5_{\mathrm{BTB}}$ show similar $\mathrm{C}_{1 / 2}$ values, close to $5.0 \mathrm{M}$ and $2.2 \mathrm{M}$, when they are denatured by means of urea and $\mathrm{GuHCl}$, respectively. The relatively low value of the ratio $\mathrm{C}_{1 / 2}($ urea $) / \mathrm{C}_{1 / 2}(\mathrm{GuHCl}) \approx$ 2.2 suggests that electrostatic interactions, that are efficiently impaired by $\mathrm{GuHCl}$, should play a limited role in the stabilization of these oligomeric structures $[23,24]$. The analysis of the thermal/chemical denaturation of these domains also highlights a number of intriguing properties, which are shared by the two domains despite their different oligomeric organization. Present data indicate that (a) thermal/chemical denaturation is a reversible process for both domains (b) thermal/chemical denaturation curves of both proteins are characterized by a single and sharp inflection point, suggestive of a cooperative two-state process, in which dissociation and unfolding are coupled; and (c) both domains maintain a significant content of secondary structure in the thermally denatured state. It is likely that the presence of a residual structure in their denatured state could favor the recovery of the native structure in the refolding process.

The ability of these domains to fold and unfold in a reversible manner is somewhat unexpected for these oligomeric assemblies. Among the few literature examples, it is worth mentioning the tetramerization domain of p53 tumor suppressor protein which shows a reversible and cooperative two-state denaturation against both temperature and $\mathrm{GuHCl}[20,25]$. This property shared by both $\mathrm{KCTD}_{\mathrm{BTB}}$ and $\mathrm{KCTD} 5_{\mathrm{BTB}}$ may have important implications for their biological function. Recent literature data have shown that the POZ/BTB domains are used by KCTDs proteins to form heteromeric associations among different members of the family [4]. It has been shown, for example, that KCTD10 interacts with KCTD13 [26] and that KCTD6 is able to form heteromeric assemblies, likely tetramers, with KCTD11 and KCTD21 [4]. Since C-terminal domains of KCTD6 and KCTD11/KCTD21 are completely unrelated and present different biological partnerships, these mixed heteroassociations by means of the POZ/BTB domain are potentially able to expand the partnership of these proteins with the possibility to create new biological routes. The capability of the POZ/BTB domain of KCTDs to fold and unfold reversibly makes the process of subunit exchange between homomeric and heteromeric assemblies easier. Indeed, given the tight association of the subunits in the tetrameric/pentameric oligomers formed by the POZ/BTB domain, these replacements are likely associated with (partial) unfolding of the involved structural subunit. The ability of these domains to refold favors the formation of a combinatorial diversity of stable complexes whose composition depends on the local concentration of the different POZ/BTB domain and on their mutual affinity.

It is intriguing to speculate that the combination observed in KCTDs of well-conserved POZ/BTB, that assures the possibility to form heteromeric associations, coupled with diversified domains may be a more general and widespread feature among oligomeric proteins that allow intricate regulations of biological processes.

\section{Abbreviations}

$\begin{array}{ll}\text { CD: } & \text { Circular dichroism } \\ \text { KCTD5 }_{\text {Bтв }}: & \text { POZ/BTB domain of KCTD5 } \\ \text { KCTD6 }_{\text {BTB }}: & \text { POZ/BTB domain of KCTD6 } \\ \text { GuHCl: } & \text { Guanidine hydrochloride. }\end{array}$

\section{Conflict of Interests}

The authors declare that there is no conflict of interests regarding the publication of this paper.

\section{Authors' Contribution}

Luciano Pirone and Carla Esposito equally contributed to this work.

\section{Acknowledgments}

This work was funded by the Italian MIUR for financial support (PRIN2009 and FIRB RBAP114AMK_006) and by the Regione Campania (Bando POR per la Realizzazione della Rete delle Biotecnologie in Campaniaprogetto FARMABIONET). The authors thank Drs Barbara Ziaco for skillful technical support and Nicole Balasco for the help in sequence analysis.

\section{References}

[1] E. D. Levy, E. B. Erba, C. V. Robinson, and S. A. Teichmann, "Assembly reflects evolution of protein complexes," Nature, vol. 453, no. 7199, pp. 1262-1265, 2008.

[2] T. Perica, C. Chothia, and S. A. Teichmann, "Evolution of oligomeric state through geometric coupling of protein interfaces," Proceedings of the National Academy of Sciences of the United States of America, vol. 109, pp. 8127-8132, 2012.

[3] P. J. Stogios, G. S. Downs, J. J. S. Jauhal, S. K. Nandra, and G. G. Privé, "Sequence and structural analysis of BTB domain proteins," Genome Biology, vol. 6, no. 10, article R82, 2005.

[4] E. De Smaele, L. Di Marcotullio, M. Moretti et al., "Identification and characterization of KCASH2 and KCASH3, 2 novel cullin 3 adaptors suppressing histone deacetylase and hedgehog activity in medulloblastoma," Neoplasia, vol. 13, no. 4, pp. 374-385, 2011.

[5] G. Canettieri, L. Di Marcotullio, A. Greco et al., "Histone deacetylase and Cullin3-REN KCTD11 ubiquitin ligase interplay regulates Hedgehog signalling through Gli acetylation," Nature Cell Biology, vol. 12, no. 2, pp. 132-142, 2010.

[6] S. Correale, L. Pirone, L. Di Marcotullio et al., "Molecular organization of the cullin E3 ligase adaptor KCTD11," Biochimie, vol. 93, no. 4, pp. 715-724, 2011.

[7] Y. Chen, Z. Yang, M. Meng et al., "Cullin mediates degradation of RhoA through evolutionarily conserved BTB adaptors to control actin cytoskeleton structure and cell movement," Molecular Cell, vol. 35, no. 6, pp. 841-855, 2009.

[8] Y. Bayón, A. G. Trinidad, M. L. de la Puerta et al., "KCTD5, a putative substrate adaptor for cullin3 ubiquitin ligases," The FEBS Journal, vol. 275, no. 15, pp. 3900-3910, 2008.

[9] F. Mei, J. Xiang, S. Han et al., "Expression, purification, and secondary structure characterization of recombinant KCTD1," Biochemistry, vol. 77, no. 8, pp. 941-945, 2012. 
[10] R. Azizieh, D. Orduz, P. van Bogaert et al., "Progressive myoclonic epilepsy-associated gene KCTD7 is a regulator of potassium conductance in neurons," Molecular Neurobiology, vol. 44, no. 1, pp. 111-121, 2011.

[11] J. Schwenk, M. Metz, G. Zolles et al., "Native GABAB receptors are heteromultimers with a family of auxiliary subunits," Nature, vol. 465, no. 7295, pp. 231-235, 2010.

[12] C. Golzio, J. Willer, M. E. Talkowski et al., "KCTD13 is a major driver of mirrored neuroanatomical phenotypes of the 16p11. 2 copy number variant," Nature, vol. 485, no. 7398, pp. 363-367, 2012.

[13] L. Di Marcotullio, G. Canettieri, P. Infante, A. Greco, and A. Gulino, "Protected from the inside: endogenous histone deacetylase inhibitors and the road to cancer," Biochimica et Biophysica Acta, vol. 1815, no. 2, pp. 241-252, 2011.

[14] P. van Bogaert, R. Azizieh, J. Désir et al., "Mutation of a potassium channel-related gene in progressive myoclonic epilepsy," Annals of Neurology, vol. 61, no. 6, pp. 579-586, 2007.

[15] L. Pirone, S. Correale, I. de Paola et al., "Design, synthesis and characterization of a peptide able to bind proteins of the KCTD family: implications for KCTD-cullin 3 recognition," Journal of Peptide Science, vol. 17, no. 5, pp. 373-376, 2011.

[16] I. S. Dementieva, V. Tereshko, Z. A. McCrossan et al., "Pentameric assembly of potassium channel tetramerization domain-containing protein 5," Journal of Molecular Biology, vol. 387, no. 1, pp. 175-191, 2009.

[17] S. Correale, C. Esposito, L. Pirone, L. Vitagliano, S. Di Gaetano, and E. Pedone, "A biophysical characterization of the folded domains of KCTD12: insights into interaction with the GABAB2 receptor," Journal of Molecular Recognition, vol. 26, pp. 488-495, 2013.

[18] S. Lange, S. Perera, P. Teh, and J. Chen, "Obscurin and KCTD6 regulate cullin-dependent small ankyrin-1 (sAnk1. 5) protein turnover," Molecular Biology of the Cell, vol. 23, pp. 2490-2504, 2012.

[19] S. Coni, L. Antonucci, D. D’Amico et al., "Gli2 acetylation at lysine 757 regulates hedgehog-dependent transcriptional output by preventing its promoter occupancy," PloS One, vol. 8, Article ID e65718, 2013.

[20] C. R. Johnson, P. E. Morin, C. H. Arrowsmith, and E. Freire, "Thermodynamic analysis of the structural stability of the tetrameric oligomerization domain of p53 tumor suppressor," Biochemistry, vol. 34, no. 16, pp. 5309-5316, 1995.

[21] F. Catanzano, C. Giancola, G. Graziano, and G. Barone, "Temperature-induced denaturation of ribonuclease S: a thermodynamic study," Biochemistry, vol. 35, no. 41, pp. 1337813385, 1996.

[22] P. J. Stogios and G. G. Privé, "The BACK domain in BTB-kelch proteins," Trends in Biochemical Sciences, vol. 29, no. 12, pp. 634637, 2004.

[23] J. K. Myers, C. N. Pace, and J. M. Scholtz, "Denaturant m values and heat capacity changes: relation to changes in accessible surface areas of protein unfolding," Protein Science, vol. 4, no. 10, pp. 2138-2148, 1995.

[24] C. E. Dempsey, T. J. Piggot, and P. E. Mason, "Dissecting contributions to the denaturant sensitivities of proteins," Biochemistry, vol. 44, no. 2, pp. 775-781, 2005.

[25] M. G. Mateu and A. R. Fersht, "Nine hydrophobic side chains are key determinants of the thermodynamic stability and oligomerization status of tumour suppressor p53 tetramerization domain," The EMBO Journal, vol. 17, no. 10, pp. 2748-2758, 1998.
[26] M. E. Sowa, E. J. Bennett, S. P. Gygi, and J. W. Harper, "Defining the human deubiquitinating enzyme interaction landscape," Cell, vol. 138, no. 2, pp. 389-403, 2009. 

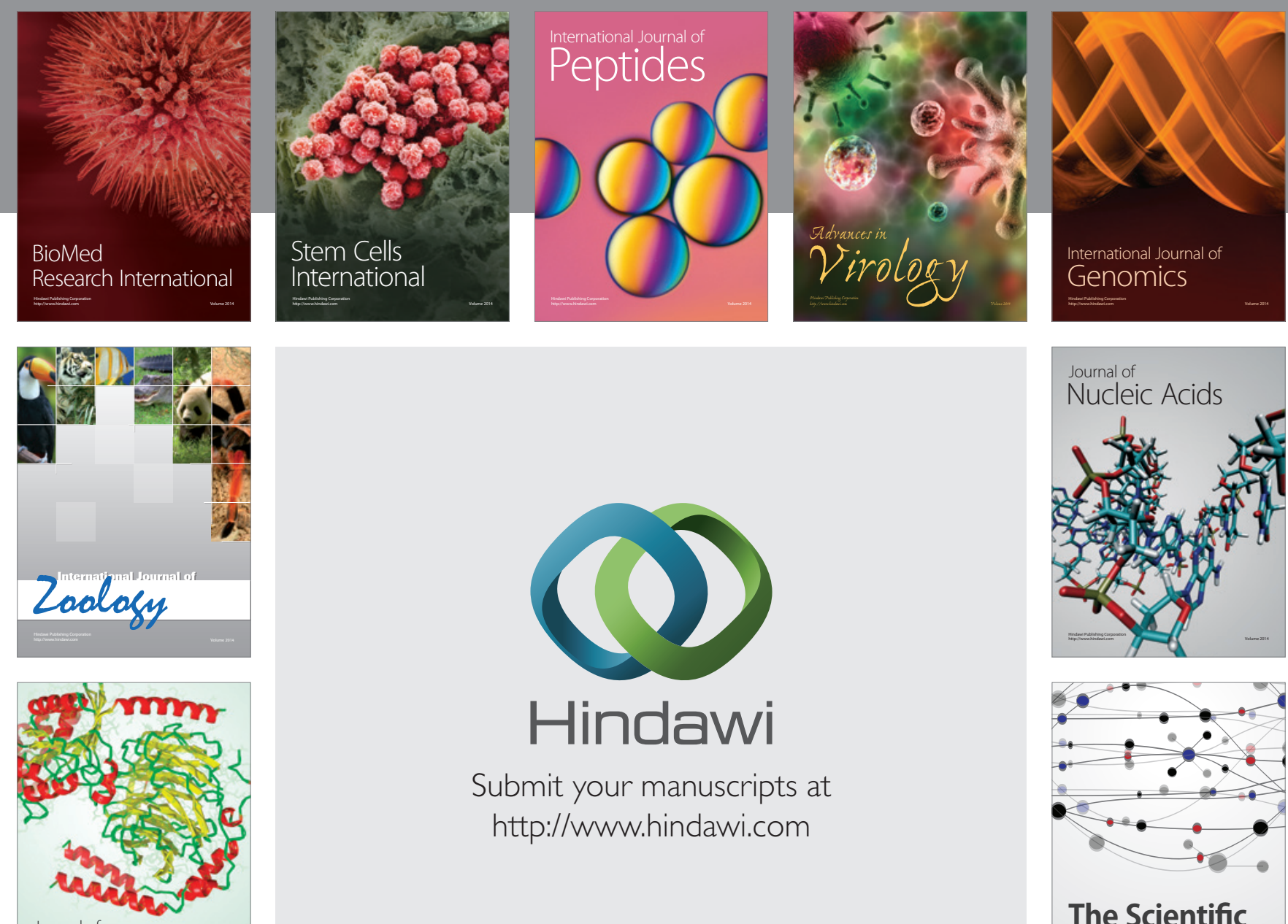

Submit your manuscripts at

http://www.hindawi.com

Journal of
Signal Transduction
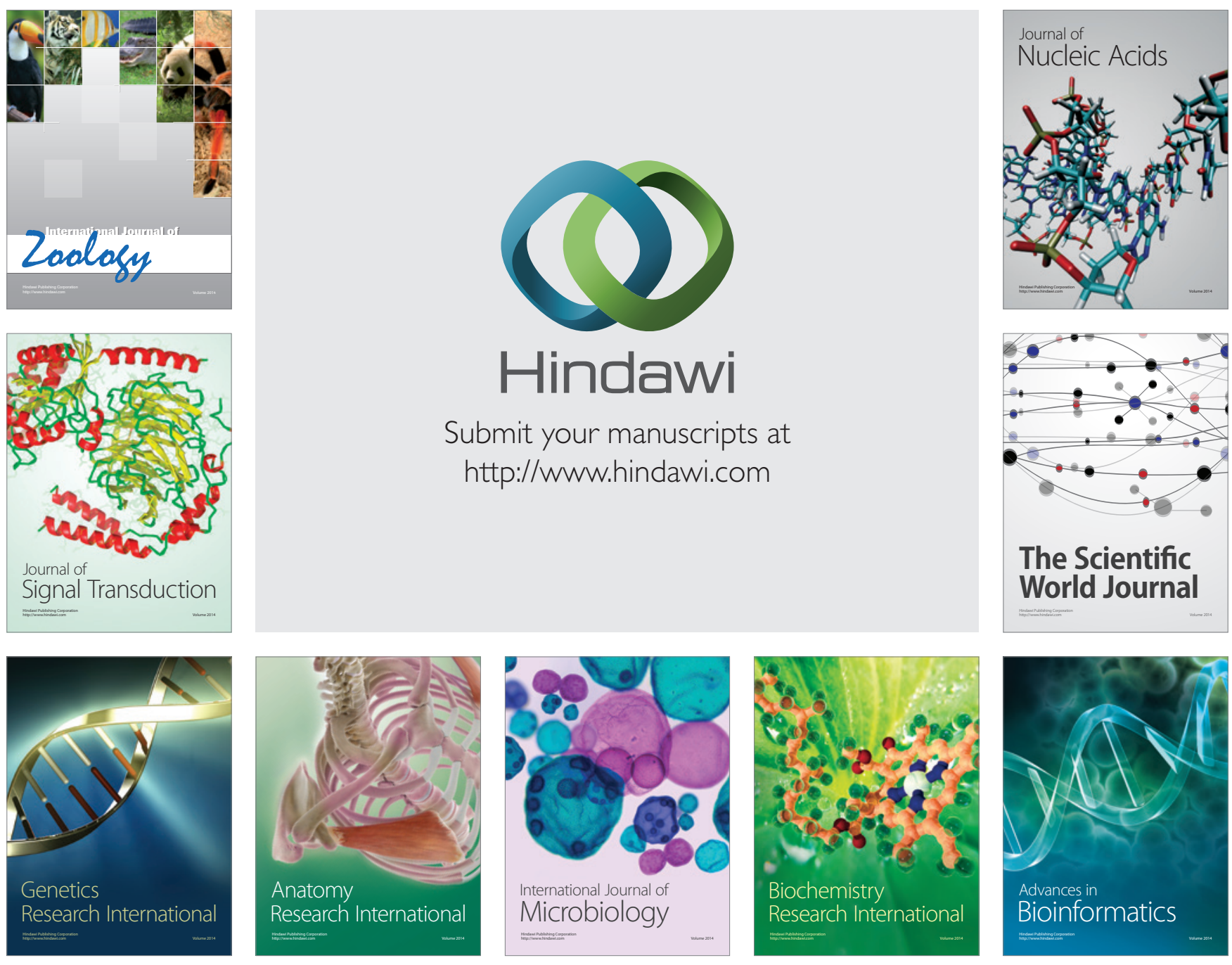

The Scientific World Journal
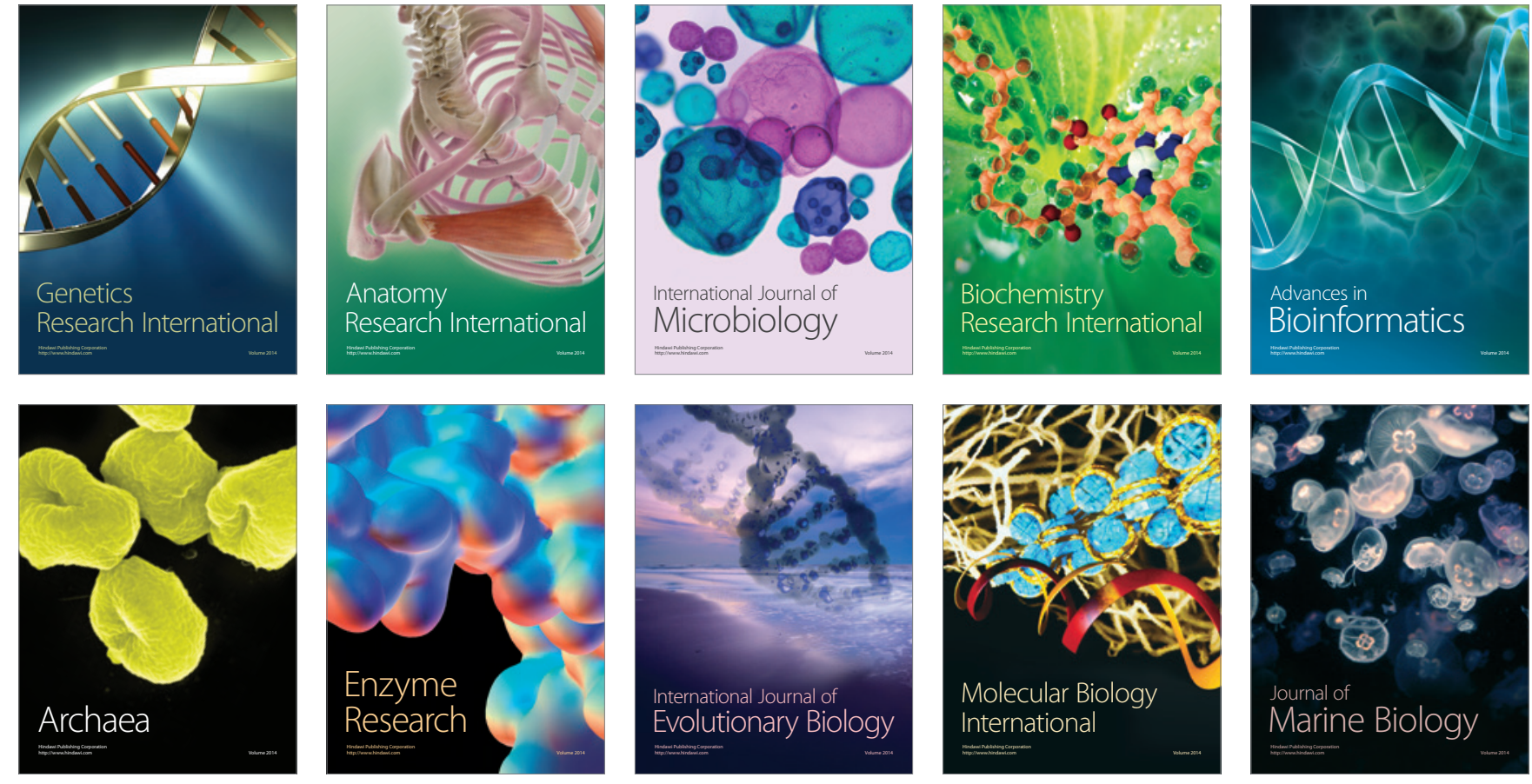